\title{
Frequency of pathogenic germline variants in BRCA1, BRCA2, PALB2, CHEK2 and TP53 in ductal carcinoma in situ diagnosed in women under the age of 50 years
}

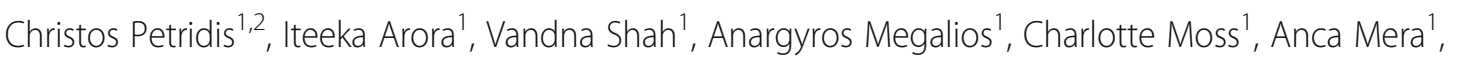
Angela Clifford', Cheryl Gillett', Sarah E. Pinder ${ }^{1}$, lan Tomlinson ${ }^{3}$, Rebecca Roylance ${ }^{4}$, Michael A. Simpson ${ }^{2}$ and Elinor J. Sawyer ${ }^{1,5^{*}}$ (i)

\begin{abstract}
Introduction: Ductal carcinoma in situ (DCIS) is a non-obligate precursor of invasive ductal breast cancer, and approximately $20 \%$ of screen-detected tumours are pure DCIS. Most risk factors for breast cancer have similar associations with DCIS and IDC; however, there is limited data on the prevalence of the known high and moderate penetrance breast cancer predisposition genes in DCIS and which women with DCIS should be referred for genetic screening.

The aim of this study was to assess the frequency of germline variants in BRCA2, BRCA1, CHEK2, PALB2 and TP53 in DCIS in women aged less than 50 years of age.

Methods: After DNA extraction from the peripheral blood, Access Array technology (Fluidigm) was used to amplify all exons of these five known breast cancer predisposition genes using a custom made targeted sequencing panel in 655 cases of pure DCIS presenting in women under the age of 50 years together with 1611 controls.

Results: Case-control analysis revealed an excess of pathogenic variants in BRCA2 (OR $=27.96,95 \% \mathrm{Cl} 6.56-119.26$, $\left.P=2.0 \times 10^{-10}\right)$ and CHEK2 $\left(\mathrm{OR}=8.04,95 \% \mathrm{Cl} 2.93-22.05, P=9.0 \times 10^{-6}\right)$, with weaker associations with PALB2 $(P=0$. 003), BRCA1 ( $P=0.007)$ and TP53 ( $P=0.02)$. For oestrogen receptor (ER)-positive DCIS the frequency of pathogenic variants was $9 \%$ under the age of 50 (14\% with a family history of breast cancer) and $29 \%$ under the age of 40 (42\% with a family history of breast cancer). For ER-negative DCIS, the frequency was $9 \%$ (16\% with a family history of breast cancer) and $8 \%$ (11\% with a family history of breast cancer) under the ages of 50 and 40 , respectively.

Conclusions: This study has shown that breast tumourigenesis in women with pathogenic variants in BRCA2, CHEK2, PALB2, BRCA1 and TP53 can involve a DCIS precursor stage and that the focus of genetic testing in DCIS should be on women under the age of 40 with ER-positive DCIS.
\end{abstract}

Keywords: Germline variants, BRCA2, CHEK2, PALB2, BRCA1, TP53, Ductal carcinoma in situ

\footnotetext{
* Correspondence: elinor.sawyer@kcl.ac.uk

'School of Cancer and Pharmaceutical Sciences, Guy's Hospital, King's

College London, London SE1 9RT, UK

${ }^{5}$ Innovation Hub, Guy's Cancer Centre, Guy's Hospital, London SE1 9RT, UK

Full list of author information is available at the end of the article
}

(c) The Author(s). 2019 Open Access This article is distributed under the terms of the Creative Commons Attribution 4.0 International License (http://creativecommons.org/licenses/by/4.0/), which permits unrestricted use, distribution, and reproduction in any medium, provided you give appropriate credit to the original author(s) and the source, provide a link to the Creative Commons license, and indicate if changes were made. The Creative Commons Public Domain Dedication waiver (http://creativecommons.org/publicdomain/zero/1.0/) applies to the data made available in this article, unless otherwise stated. 


\section{Introduction}

Ductal carcinoma in situ (DCIS) is considered a non-obligate precursor of invasive breast cancer of ductal/no special type (IDC) as many IDCs have evidence of associated DCIS at presentation $[1,2]$ and the two components have similar genetic changes, suggesting that in the majority of cases the invasive component has arisen from the DCIS [3]. Synchronous DCIS is more frequent in luminal and HER2-positive IDC (53\% and 63\%, respectively) than invasive basal breast cancer (33\%) [4]. Since the introduction of screening mammography, there has been an increase in the reported incidence of pure DCIS with no invasive component [5], with about $20 \%$ of screen-detected tumours being pure DCIS [6].

Most non-genetic risk factors for breast cancer have similar associations with DCIS and IDC, again supporting the notion that DCIS is a precursor of invasive cancer $[7,8]$. Epidemiological studies have shown there is an inherited predisposition to DCIS, with women with DCIS being 2.4 times more likely to have an affected mother and sister with breast cancer than controls [9]. One study of almost 40,000 women suggested that the familial relative risk of DCIS may be greater than that of invasive breast cancer [10], but this was not confirmed in the Million Women Study, which showed a similar association with family history for DCIS and IDC [8].

The familial risk associated with invasive breast cancer is in part explained by both high-risk rare variants and low-risk susceptibility loci. We have shown that the majority of low-risk invasive breast cancer predisposition loci also predispose to DCIS and, as for invasive disease, different loci predispose to ER-positive and ER-negative DCIS and high and low grade DCIS [11]. However, the frequency of pathogenic high- and moderate-risk variants in DCIS is not clear. Claus et al. studied 369 women (mean age 53.8 years) with pure DCIS selected from a case-control study of carcinoma in situ and found that $2.4 \%$ had pathogenic variants in $B R C A 2$ and $0.8 \%$ in BRCA1 [12]. Hall et al. analysed a highly selected cohort of women with carcinoma in situ (LCIS and DCIS) that were referred to Myriad for genetic testing. They found that $5.2 \%$ of women with pure carcinoma in situ (CIS) had BRCA1/2 mutations (2.3\% if women with a family history of breast cancer were excluded) and like Claus et al. that BRCA2 mutations were more common than $B R C A 1$ [13]. Both these studies were performed before gene panel genetic testing was available, and therefore, other breast cancer predisposition genes such as PALB2, CHEK2 and TP53 were not assessed.
In this study, we report the frequency of rare variants in five known breast cancer predisposition genes (BRCA2, BRCA1, TP53, CHEK2 and PALB2) in 655 cases of pure DCIS with no invasive disease in women diagnosed before the age of 50. These cases were included in our previous study of low-risk susceptibility loci in DCIS [11].

\section{Methods}

\section{Samples}

Six hundred fifty-five cases of pure DCIS with no invasive disease diagnosed in women aged under 50 were included in this study, Table 1 . The majority of cases (633) were recruited through the ICICLE study (MREC 08/ H0502/4) from 95 hospitals throughout the UK. This study was set up with the specific aim of investigating genetic predisposition to DCIS in the UK. A further 22 cases were recruited through the King's Health Partners (KHP) Cancer Biobank (NHS REC ref. 12-EE-0493). Samples from patients under the age of 50 were selected for this analysis in order to enrich for cases likely to have a genetic component to their disease.

All controls were collected through the ICICLE and GLACIER studies (a similar study of lobular breast cancer, MREC 06/Q1702/64) and were identified by asking patients (cases) from both studies at the time of recruitment to identify female non-blood relatives or friends who were willing to donate a blood sample. These healthy volunteers were only eligible if they had no personal or family (up to second degree) history of invasive breast cancer, lobular carcinoma in situ (LCIS), DCIS or benign breast disease. Controls were not age matched and could be of any age, although older individuals were preferred (super-controls), as they had lived through many of their at-risk years.

All participants (cases and controls) donated a blood sample and were asked to complete a self-administered paper-based questionnaire on their family history.

Data on grade and oestrogen receptor (ER) status was ascertained mostly from the hospital pathology reports. In 200 cases where the grade data was missing from the report but a tumour block was available, a H\&E section was cut and the DCIS was graded by the study histopathologist (SEP) according to UK and CAP guidelines [14]. A subset of 81 cases, graded in the pathology report and with a tumour block available, was examined to assess the reliability of the cytonuclear grade provided

Table 1 ER status and cytonuclear grade of DCIS by age

\begin{tabular}{|c|c|c|c|c|c|c|c|c|}
\hline Age & Total & $\mathrm{ER}+$ & ER- & ER unknown & High grade & Int grade & Low grade & Grade unknown \\
\hline $40-49$ & 555 & $345(62 \%)$ & $62(11 \%)$ & $148(27 \%)$ & $345(62 \%)$ & $148(27 \%)$ & $47(8 \%)$ & $15(3 \%)$ \\
\hline$<40$ & 100 & 45 (45\%) & $26(26 \%)$ & 29 (29\%) & 72 (72\%) & $20(20 \%)$ & $7(7 \%)$ & $1(1 \%)$ \\
\hline All & 655 & 390 & 88 & 177 & 417 & 168 & 54 & 16 \\
\hline
\end{tabular}


by the pathology reports. In the majority of cases (86.5\%), grade was concordant with the pathology report. Nine cases were re-graded as low/intermediate grade and two cases as high grade. As the study pathologist re-graded the samples on a single $H \& E$ section, rather than all the blocks from an individual case, and in some cases on re-excision specimens with residual disease rather than the original excision specimen, the grade reported in the pathology report, if available, was used for the purposes of this study.

If ER status was not available from the local histopathology report and the tumour block was available, immunohistochemistry was performed and scored using the Allred method as previously described [11]. An Allred score of 3 or more was considered ER+ and that with scores of $0-2$ (approximately equivalent to less than $1 \%$ of nuclei) were regarded as ER-.

\section{Next-generation sequencing}

After DNA extraction from the peripheral blood, Access Array technology (Fluidigm) was used to amplify all exons of BRCA2, BRCA1, TP53, CHEK2 and PALB2 using a custom made targeted sequencing panel consisting of 321 amplicons (Additional file 1). The Fluidigm designed primers were supplied in single-plex with forward and reverse primers combined; these were multiplexed according to supplier's instruction to achieve optimal efficiency. Purified libraries were quantified using Qubit High Sensitivity Assay Kit, and their average length size was measured in Tapestation using the D1000 screentape. The quantity and length size values obtained from the readings were used to calculate the final Molar concentration in order to prepare each sequencing library at $4 \mathrm{nM}$ containing 960 samples, based on the following formula:

$$
\begin{aligned}
& \text { Molar Concentration }(\text { in } \mathrm{nM}) \\
& =\text { Concentration }(\text { in } \mathrm{ng}) \times 10^{6} \times\left(\frac{1}{649}\right) \times\left(\frac{1}{\text { average size }(\text { in bp })}\right)
\end{aligned}
$$

All quantified libraries were subsequently sequenced on a HiSeq2500 (Illumina).

\section{Bioinformatics analysis}

Primer sites from the amplicons were trimmed using Btrim, and then, sequences were aligned to the reference genome (http://www.novocraft.com, GRCh37 version) using Novoalign (Gap opening penalty $=65$ and gap extension penalty $=7$ thresholds were applied). Picard tools (v1.74 https://github.com/broadinstitute/picard) and Bedtools (v2.17.0) were used to assess coverage. Variant calling was performed using Samtools and annotated using the Annovar tool [15]. We optimised the calling based on a set of variants that were positive controls, and Samtools was the optimal caller compared to
HaplotypeCaller from GATK. The transcript that was used for each gene is reported in Additional file 1. The frequency of variant alleles from European reference populations was obtained from three sources (1000 genomes, ESP, ExAC), Additional file 2.

Variants were further filtered based on read depth, quality score, and genotypic quality. All variants with a read depth $<10$, quality score $<20$, or genotypic score $<$ 20 were excluded from the analysis. In addition, variants with an allelic ratio $<0.2$ were excluded irrespective of read depth and variants with an allelic ratio $<0.3$ and read depth $<50$ were also removed.

Variants that had been previously clinically evaluated and deposited in the ClinVar database (https://www. ncbi.nlm.nih.gov/clinvar/) were assigned labels of benign, variants of unknown significance (VUS), conflicting or pathogenic, as per ClinVar.

Variants not present in the ClinVar database were considered pathogenic if they were predicted to lead to protein truncation (frameshift indels, stop-gain, stop-loss or intronic variants within two base pairs of the splicing junction; Additional file 3) and variants of unknown significance (VUS) if they were nonsynonymous substitutions or in-frame indels. Novel variants in the last exon of a gene meeting the above criteria of pathogenic were not excluded as, although unlikely to result in loss of function through nonsense mediated decay, these variants may have substantial impact on the protein product. However, no such variants were detected in this study.

To further investigate the importance and validity of our findings, we used an external resource of controls. A non-Finnish European population of controls from gno$\mathrm{mAD}$ (gnomAD controls v2.1) was used as a replication control cohort.

\section{Statistical analysis}

Fisher's exact test was used for gene based rare variant analysis for both case-control and case-only analyses. One sided test was selected since the expectation was enrichment rather than deficit of variants in cases over controls. No adjustments have been made to account for multiple testing. With the current sample size, we have $\sim 80 \%$ power (alpha $=0.05$ ) to detect variants of combined allele frequency $=0.001$ and an effect size of OR $=5$.

\section{Validation}

All putative pathogenic alleles identified by the above methods were confirmed by Sanger sequencing.

\section{Assessment of copy number variation}

Two bioinformatics tools were used to assess copy number variation $(\mathrm{CNV})$ in our sequencing data: CNVkit 
version 0.9 .5 [16] and ONCOCNV version 6.9 [17]. The first, CNVkit, running in amplicon sequencing mode, was used to identify exon-level copy number variations using on-target coverages alone. One thousand six hundred eleven control samples were used to construct a reference copy number profile, and the default circular binary segmentation (CBS) algorithm was used to derive segments. The second, ONCOCNV, is a package specifically designed for amplicon sequencing data. Fifteen randomly selected control samples were used to construct the reference copy number profile, and the default cghseg algorithm was used to derive segments. All CNVs detected by these methods underwent multiplex ligation-dependent probe amplification (MLPA) using MRC Holland kits (https://www.mlpa.com/) in order to validate the finding. DNA samples were amplified, and PCR products were analysed on the ABI 3730 Genetic Analyser (Applied Biosystems) using TAMRA 500, as per the manufacturer's instructions. Coffalyser (MRC Holland) was used to call exonic deletions/rearrangements.

\section{Results}

The analysis was performed on 655 cases of pure DCIS with no invasive disease diagnosed in women aged under 50, together with 1611 controls. The median age of cases was 45 years (interquartile range 6) and of controls was 52 (interquartile range 12). Data on grade and oestrogen receptor (ER) status were available for $98 \%$ and $73 \%$ of the cases in the study, respectively, Table 1.

The mean coverage of our target region was 800 reads across all samples, with an average of at least 40 reads for more than $98 \%$ of the target region per sample. Of the 321 amplicons analysed, seven failed to amplify consistently across the majority of the samples; however, even for these seven the majority of samples had at least 10 reads for $90 \%$ of the amplicon, and there was no difference in amplification between cases and controls, Additional file 4. No exon-level CNVs were identified using CNVkit version 0.9.5. Thirteen copy number variants across eight samples were detected by ONCOCNV; however, none were confirmed using MLPA, Additional file 5.
We found an association with DCIS and pathogenic variants in BRCA2, CHEK2, PALB2, BRCA1 and TP53, Table 2 (individual raw data, Additional file 2).

\section{BRCA2}

The strongest association was with $B R C A 2(\mathrm{OR}=27.96$, 95\%CI 6.56-119.26, $P=2 \times 10^{-10}$ ), Table 2, Fig. 1a. Of the 22 pathogenic variants identified, all had been previously described apart from a novel frameshift in exon 11 (c.5754dupT:p.H1918fs). Fifty percent of pathogenic variants occurred in exon 11. Only two pathogenic variants occurred in more than one patient: exon20:c.8575delC:p.Q2859fs in two patients and exon25:c.C9382T:p.R3128X in two patients, Additional file 6. Ninety-five percent of the cases with a pathogenic variant in $B R C A 2$ had high or intermediate grade DCIS, and in the 15 cases where ER status was known, all were ER positive. There was also an association with age < 40 years $(\mathrm{OR}=4.12,95 \% \mathrm{CI} 1.75-9.69, P=0.003)$, Table 3 , and family history of breast cancer in a first-degree relative $(\mathrm{OR}=4.29,95 \% \mathrm{CI} 1.82-10.08, P=0.001)$, Table 4 .

\section{CHEK2}

CHEK2 also showed a strong association with DCIS $\left(\mathrm{OR}=8.04,95 \% \mathrm{CI} 2.93-22.05, P=9 \times 10^{-6}\right)$, but with a smaller effect size than $B R C A 2$, Table 2, Fig. 1b. Of the 16 pathogenic variants detected, 13 were the c.1100delC mutation. The remaining three variants were novel frameshift mutations, one in exon 3: c.401_402del, and two in exon 12: c.1262delT and c.1368dupA, Additional file 7 . Again, all were high or intermediate grade DCIS and, of the 14 with known ER status, 12 were ER positive. There was no association with age $<40$ ( $81 \%$ of cases occurred in the 40-49 age group) or family history, Tables 3 and 4 .

\section{PALB2}

There was an association with PALB2 (OR 14.88, 95\%CI $1.79-123.88, P=0.003)$, Table 2 , but this was weaker than that for $B R C A 2$ and $C H E K 2$. Of the six pathogenic $P A L B 2$ variants detected, three were novel frameshifts

Table 2 Association of known pathogenic variants and DCIS in women < 50 years of age by gene. For the GnomAD comparison, Non-Finnish European controls were used (v2.1)

\begin{tabular}{|c|c|c|c|c|c|c|c|}
\hline Gene & $\begin{array}{l}\text { Pathogenic variants } \\
\text { in cases }(N=655)\end{array}$ & $\begin{array}{l}\text { Pathogenic variants in } \\
\text { controls }(N=1611)\end{array}$ & OR (95\% Cl) & $P$ value & $\begin{array}{l}\text { Pathogenic variants } \\
\text { in gnomAD controls } \\
(N=21,384)\end{array}$ & $\begin{array}{l}\text { OR (95\% Cl) } \\
\text { (vs gnomAD controls) }\end{array}$ & $\begin{array}{l}P \text { value } \\
\text { (vs gnomAD controls) }\end{array}$ \\
\hline$\overline{B R C A 2}$ & $22(3.4 \%)$ & $2(0.1 \%)$ & $\begin{array}{l}27.96(6.56- \\
119.26)\end{array}$ & $\begin{array}{l}2 \times \\
10^{-10}\end{array}$ & $76(0.35 \%)$ & $9.74(6.02-15.76)$ & $1 \times 10^{-13}$ \\
\hline CHEK2 & $16(2.4 \%)$ & $5(0.3 \%)$ & $8.04(2.93-22.05)$ & $9 \times 10^{-6}$ & $144(0.67 \%)$ & $3.69(2.19-6.23)$ & $2 \times 10^{-5}$ \\
\hline PALB2 & $6(0.9 \%)$ & $1(0.06 \%)$ & $\begin{array}{l}14.88(1.79- \\
123.88)\end{array}$ & 0.003 & $22(0.1 \%)$ & $8.98(3.63-22.21)$ & $1 \times 10^{-4}$ \\
\hline$B R C A 1$ & $4(0.6 \%)$ & $0(0 \%)$ & inf & 0.007 & 81 (0.38\%) & $1.62(0.59-4.42)$ & 0.32 \\
\hline TP53 & $3(0.5 \%)$ & $0(0 \%)$ & $\inf$ & 0.02 & $16(0.07 \%)$ & $6.14(1.79-21.14)$ & 0.018 \\
\hline
\end{tabular}



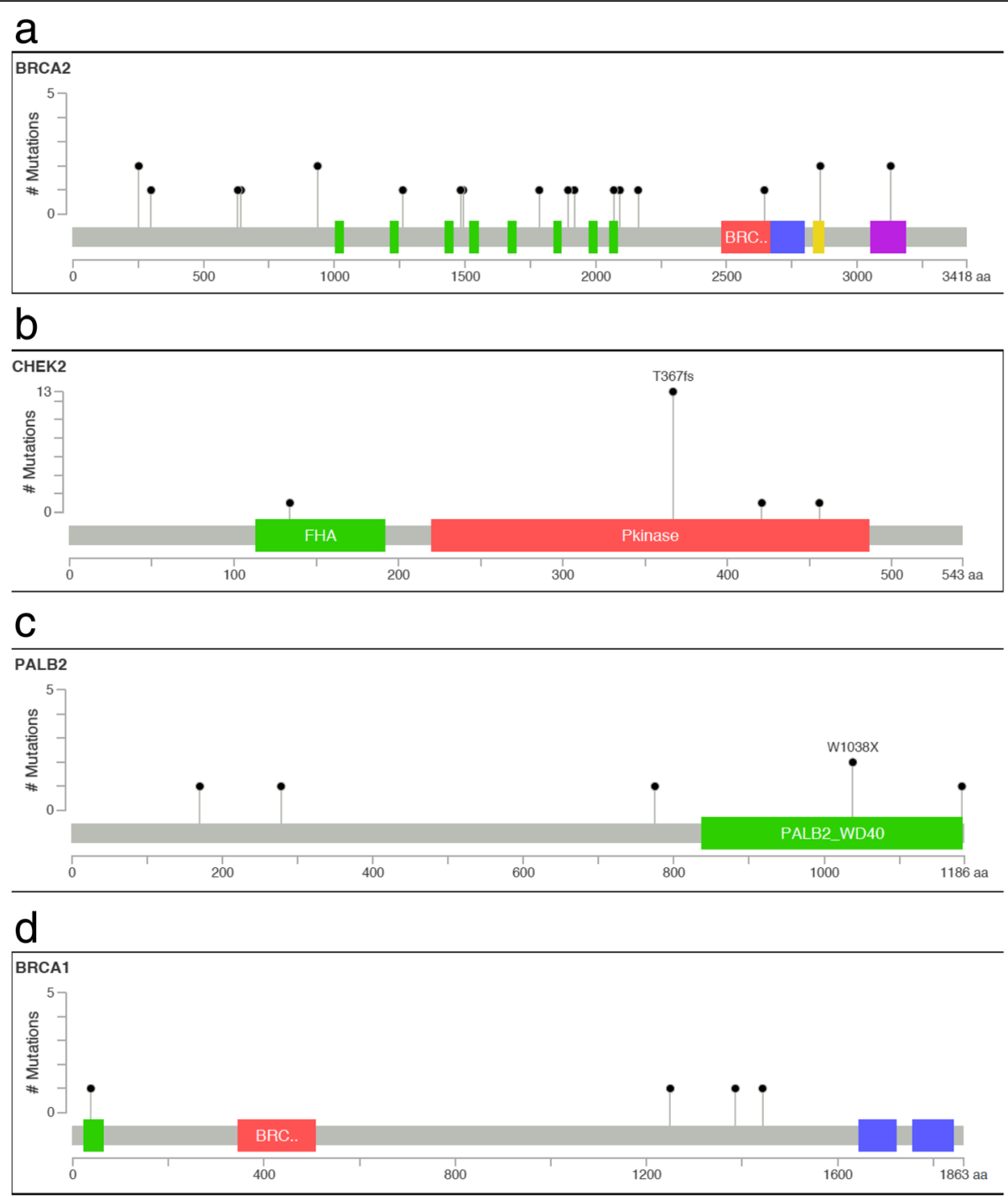

Fig. 1 Position of pathogenic variants in DCIS cases in a BRCA2, b CHEK2, c PALB2, d BRCA1

(two in exon 4, one in exon 5, Fig. 1c, Additional file 8). Of the three known, two were in exon 10 (rs180177132), which has previously been shown to be associated with breast cancer $(\mathrm{OR}=4.21,95 \% \mathrm{CI} 1.85-9.61)$, but with no evidence of a differential association with ER status [18]. All women with $P A L B 2$ pathogenic variants had high or intermediate grade DCIS and were ER positive. There was no association with age $<40$, but there was an association with family history of breast cancer in a first-degree relative $(\mathrm{OR}=14.37,95 \% \mathrm{CI} 1.68-123.25, P$ $=0.006$ ), Table 4 .

\section{BRCA1}

There were four DCIS cases that were found to harbour pathogenic BRCA1 germline variants and no controls $((P=0.007)$, Table 2 , Additional file 9 . One was the

Table 3 Case only analysis of pathogenic variants in DCIS by age

\begin{tabular}{lllll}
\hline Gene & Pathogenic variants in cases aged $<40(N=100)$ & Pathogenic variants in cases aged $40-49(N=555)$ & OR $(95 \%$ Cl $)$ & $P$ value \\
\hline BRCA2 & $9(9 \%)$ & $13(2.3 \%)$ & $4.12(1.75-9.69)$ & 0.003 \\
CHEK2 & $3(3 \%)$ & $13(2.3 \%)$ & $1.29(0.37-4.54)$ & 0.72 \\
PALB2 & $1(1 \%)$ & $5(0.9 \%)$ & $1.11(0.13-9.53)$ & 1 \\
BRCA1 & $1(1 \%)$ & $3(0.5 \%)$ & $1.86(0.19-17.90)$ & 0.485 \\
TP53 & $1(1 \%)$ & $2(0.4 \%)$ & $2.79(0.25-30.86)$ & 0.39 \\
\hline
\end{tabular}


Table 4 Case only analysis of pathogenic variants in DCIS for family history of breast cancer

\begin{tabular}{|c|c|c|c|c|}
\hline \multirow[t]{2}{*}{ Gene } & \multicolumn{2}{|c|}{ Carriers with $\mathrm{FH}$ of $\mathrm{BC}$ in first degree relative vs no $\mathrm{FH}$} & \multicolumn{2}{|c|}{ Carriers with $\mathrm{FH}$ of $\mathrm{BC}$ in any relative vs no $\mathrm{FH}$} \\
\hline & $\mathrm{OR}(95 \% \mathrm{Cl})$ & $P$ & OR $(95 \% \mathrm{Cl})$ & $P$ \\
\hline BRCA2 & $4.29(1.82-10.08)$ & 0.001 & $4.90(1.65-14.54)$ & 0.002 \\
\hline CHEK2 & $0.93(0.30-2.90)$ & 1 & $1.76(0.64-4.86)$ & 0.32 \\
\hline PALB2 & $14.37(1.68-123.25)$ & 0.006 & $5.27(0.61-45.20)$ & 0.12 \\
\hline BRCA1 & $8.52(0.88-82.07)$ & 0.058 & $3.14(0.33-30.26)$ & 0.36 \\
\hline TP53 & $1.40(0.13-15.47)$ & 1 & $2.09(0.19-23.07)$ & 0.62 \\
\hline
\end{tabular}

$F H$ family history, $B C$ breast cancer

well-described exon12:c.C4327T:p.R1443X, and the other three were novel frameshift mutations, two in exon 11 and one in exon 3. Three were ER negative (one unknown) and three high-grade DCIS. There was no association with age $<40$ and a borderline association with family history of breast cancer in a first-degree relative $(P=0.058)$. Three of the cases had a family history of breast and or ovarian cancer in first-degree relatives under the age of 50 . However, the case with the novel frameshift variant in exon 10: c.3750delG:p.E1250fs had a strong family history of other cancers (cervical, lung, and oral) but not breast.

\section{TP53}

There were three DCIS cases that were found to harbour pathogenic TP53 germline variants and no controls ( $(P$ $=0.02$ ), Table 2 . Two were stopgain variants (one novel), and the other a nonsynomous variant (rs397514495) considered pathogenic in the literature [19], but recently suggested to be a VUS as it does not disrupt all the functions of TP53 just apoptosis [20], Additional file 10a. The women that carried these variants did not meet the criteria for classic Li-Fraumeni or Li-Fraumeni-like syndrome. The novel stopgain variant (NM_000546:exon4:c.G272A:p.W91X) was found in a woman with bilateral DCIS at age 35 and a first-degree relative with breast cancer $<40$ years, but no other cancers. The other known stopgain variant was identified in a woman with DCIS at age 40 whose father developed an unknown cancer at age 50 but with no other family history of cancer. The missense variant occurred in a woman with DCIS at age 45 and a family history of breast cancer in a second-degree relative aged 60, but no other cancers. Again, all had intermediate or high-grade DCIS, with two ER positive and two ER negative (in the bilateral case one side was ER positive and the other ER negative).

As most pathogenic germline mutations in TP53 are missense rather than truncating mutations [21], it is possible that by only considering novel variants as pathogenic if they were predicted to lead to protein truncation we may have missed novel pathogenic missense variants in TP53. We therefore looked at these in more detail.
Only one novel missense variant was detected, NM_000546:exon10:c.G1054 T:p.D352Y, and this was in a control. Three variants called as VUS in the ClinVar database were identified in cases, Additional file 10b. One NM_000546:exon8:c.G869A:p.R290H is listed in the IARC TP53 database [22] as being associated with Li-Fraumeni-like syndrome and, in our study, was found in one case and two controls.

\section{Biallelic mutation carriers}

There were four patients carrying two pathogenic variants in different genes. All had a family history of cancer and three a family history of breast cancer, Additional file 11.

\section{Bilateral and subsequent invasive disease}

Metachronous bilateral disease was most commonly found in CHEK2 mutation carriers with 5/16 (31\%) developing subsequent contralateral disease after a diagnosis of unilateral DCIS (in two, this was in the form of an invasive disease, in two LCIS and in one DCIS) compared to $2 / 22$ (9\%) for $B R C A 2$. The latter, however, will be influenced by the fact that nine of the $B R C A 2$ carriers elected for bilateral prophylactic mastectomies based on their family history or the results of genetic testing (five underwent genetic testing). As this study does not have long-term follow-up data, it was not possible to assess whether germline pathogenic variants were associated with subsequent invasive recurrence of DCIS.

\section{Variants of unknown significance}

Analysis of variants of unknown significance (VUS), either known or novel, revealed no excess of VUS in $B R C A 2$, PALB2, TP53 or BRCA1, Additional file 12. A VUS in exon 4 of CHEK2 (rs77130927, c.C538T:p.R180C), which we have previously shown to have a borderline association with invasive lobular cancer (ILC) $(P=0.03$, Petridis et al, accepted Cancer Epidemiology, Biomarkers \& Prevention), was found in two DCIS cases and one control.

\section{Discussion}

Early data on women with BRCA1 and BRCA2 mutations suggested that presentation as pure DCIS was infrequent. However, Yang et al. showed that $\sim 60 \%$ of 
$B R C A 1$ - and BRCA2-associated invasive tumours had associated DCIS of similar phenotype [23]. They also found that the number of pure DCIS cases was similar in BRCA1 and BRCA2 mutations carriers (21\% vs $23 \%$ ), in contrast to Krammer et al. who reported that pure DCIS was more frequent in $B R C A 2$ mutation carriers compared to $B R C A 1$ carriers $(5 \%, 36 / 246$, versus $9 \%, 23 /$ $250, P=0.0026)$ [24]. In our study of sporadic pure DCIS, pathogenic $B R C A 2$ variants were far more common than BRCA1 mutations (3.5\% vs $0.6 \%$ ). This is similar to the data of Claus et al. [12] who found that $2.4 \%$ had pathogenic variants in $B R C A 2$ and $0.8 \%$ in $B R C A 1$ in a slightly older group of DCIS patients (mean age 53.8 years). Similarly, Hall et al. found that $5.2 \%$ of women under 50 years of age with carcinoma in situ (LCIS and DCIS) had BRCA1/2 mutations, with BRCA2 mutations being more common than BRCA1 [13].

We found that BRCA2 mutations occurred in $2.4 \%$ of DCIS in women under the age 50 and in $9 \%$ under the age of 40. All but one of these variants had been previously described in invasive breast cancer. Of the genes studied, $B R C A 2$ was the only gene where pathogenic mutations were associated with younger age. All the cases of DCIS in BRCA2 carriers were ER positive (where ER status was known), unlike invasive disease where only $77 \%$ are ER positive [25]. In contrast, BRCA1 pathogenic variants were infrequent, four in total (only one had been previously described), and associated with predominantly ER negative DCIS.

Pathogenic variants in CHEK2 were the second most common set of mutations after BRCA2 and occurred in $2.5 \%$ of pure DCIS under the age of 50 . Unlike $B R C A 2$, there was no association with age and the majority were the well-described c.1100delC variant. There was no evidence of an association with the rare missense variant p.I157T (c.T470C, rs17879961), which was found in three controls and no cases. This high frequency of CHEK2 variants in pure DCIS has not been previously described, although Schmidt et al. noted in their study of tumour characteristics in CHEK2 c.1100delC carriers that carriers from population- and hospital-based studies more often developed in situ tumours (LCIS and DCIS) compared to carriers from familial or clinical genetics center-based studies; this was interpreted as a bias estimate due to differential recruitment related to family history of breast cancer and screening [26]. Our findings are also supported by Couch et al. who reported data on the frequency of CHEK2 mutations in a series of breast cancer with and without pure DCIS allowing one to determine mutation rates in pure DCIS cases. In that study, $2.87 \%$ of DCIS cases had pathogenic CHEK2 variants compared to $1.43 \%$ in invasive disease [27].

The tumour phenotypes associated with PALB2 tumours are very similar to those associated with $B R C A 2$ tumours, with $61 \%$ of invasive tumours having associated DCIS [28]. It is therefore not surprising that we have found PALB2 pathogenic mutations in women with pure DCIS and that they are more common in women with a first-degree relative with breast cancer. However, unlike $B R C A 2$, they were not associated with age $<40$ years, this is supported by the findings of Antoniou et al. who showed there was a constant relative risk, irrespective of age, for pathogenic variants in PALB2 [29]. The frequency of $P A L B 2$ variants in our data is supported by the study by Couch et al. where one can infer from the supplementary data that the frequency of pathogenic PALB2 variants in DCIS is $0.96 \%$ [27].

Pure DCIS (73\% HER2 positive, 55\% ER positive) and high-grade comedo DCIS have been described in Li-Fraumeni Syndrome [30, 31] but our data show that TP53 mutations are infrequent in sporadic DCIS. Although two of three pathogenic variants identified had been previously described, none of the women had a family history of cancer to suggest Li-Fraumeni Syndrome (LFS).This may be because they are de novo mutations or because two were loss of function mutations which often do not have such a typical LFS phenotype as dominant negative missense mutations [32]. The known missense mutation detected has been shown to be associated with Li-Fraumeni-like syndrome rather than true LFS [22].

The odds ratios presented in this study are higher than those previously reported for these genes in invasive disease. This is likely due to the size of the study which is too small to yield stable estimates of associations with DCIS, but does give useful estimates of prevalence and, of note, is twice as large as the study by Claus et al., the only other study documenting the prevalence of BRCA1/ 2 mutations in sporadic DCIS. The other reason is due to the use of older controls. When we compare our cases to 21,384 non-Finnish European controls from gnomAD (gnomAD controls v2.1, http://gnomad.broadinstitute.org), we see that the odds ratios fall to similar levels previously reported for invasive cancer with the exception of CHEK2 (OR = 3.69, 95\%CI.19-6.23) which still remains higher than that reported in studies of invasive breast cancer $(\mathrm{OR} \sim 2)$, Table 2 . Schmidt et al. also found this in their large series from the Breast Cancer Association Consortium (invasive: $\mathrm{OR}=2.4,95 \% \mathrm{CI}$ 2.04-2.82, in situ: $\mathrm{OR}=3.53,95 \% \mathrm{CI} 2.38-5.23$ ) [26]). We also found a similar finding in lobular cancer where LCIS had a stronger association with CHEK2 mutations than ILC (ILC OR $=4.29,95 \%$ CI 1.60-11.51, $P=0.0017$; LCIS OR $=9.95,95 \%$ CI 3.44-28.82, $P=5 \times 10^{-5}$, Petridis et al. accepted Cancer Epidemiology, Biomarkers \& Prevention). This suggests that CHEK2 pathogenic variants may be predisposing to the in situ stage of breast cancer with some not progressing to the invasive state. 
Table 5 Frequency of pathogenic variants in ER-negative DCIS by age

\begin{tabular}{|c|c|c|c|c|}
\hline Gene & $\begin{array}{l}\text { Frequency under } 40 \text { years } \\
N=26\end{array}$ & $\begin{array}{l}\text { Frequency under } 40 \text { years with FH } \\
N=9\end{array}$ & $\begin{array}{l}\text { Frequency under } 50 \text { years } \\
N=89\end{array}$ & $\begin{array}{l}\text { Frequency under } 50 \text { years with FH } \\
N=38\end{array}$ \\
\hline BRCA2 & $0(0 \%)$ & $0(0 \%)$ & $0(0 \%)$ & $0(0 \%)$ \\
\hline CHEK2 & $1(3.8 \%)$ & $0(0 \%)$ & $3(3.3 \%)$ & $2(5.2 \%)$ \\
\hline PALB2 & $0(0 \%)$ & $0(0 \%)$ & $0(0 \%)$ & $0(0 \%)$ \\
\hline$B R C A 1$ & $0(0 \%)$ & $0(0 \%)$ & $3(3.3 \%)$ & $3(7.9 \%)$ \\
\hline TP53 & $1(3.8 \%)$ & $1(11 \%)$ & $2(2.2 \%)$ & $1(2.6 \%)$ \\
\hline Total (\% of ER- cases) & $2(8 \%)$ & $1(11 \%)$ & $8(9 \%)$ & $6(16 \%)$ \\
\hline
\end{tabular}

$\mathrm{FH}$ family history of breast cancer in any relative

In a study of 6478 patients with invasive breast cancer under the age of 50, Schmidt et al. [33] found a higher frequency of BRCA1 mutations compared to our study of DCIS (3.2\% versus $0.6 \%$ ). We believe that this difference in frequency of BRCA1 mutations stems from the fact that the vast majority of the samples in our study are ER+ and only $13 \%$ of the samples are ER-, compared to $25 \%$ in the invasive study of Schmidt et al.

There is currently a debate as to the need for mutation screening in women with DCIS. In this study, $7.2 \%$ of women with DCIS (irrespective of ER status) under the age of 50 had pathogenic variants in one of five known breast cancer predisposition genes. This level does not reach the current UK threshold for genetic testing (https://www.nice.org.uk/guidance/cg164/chapter/

Recommendations\#genetic-testing); however, women under 40 years of age had a $13 \%$ (11\% excluding CHEK2 variants) probability of having a germline mutation which does reach the UK threshold of $10 \%$ for routine testing. For women under 40 years of age with a family history of breast cancer, the frequency of germline mutations increases to $21 \%$.

There has been particular focus on ER-negative DCIS and whether these women should undergo HER2 testing and, if this is also negative, be offered BRCA1 and $B R C A 2$ testing, as is recommended for those with triple-negative invasive breast cancer. Unfortunately, in our series, we did not have data on HER2 receptor status as it is not routinely assessed in cases of DCIS in present clinical practice. However, looking solely at ER-negative DCIS, only $9 \%$ under the age of 50 and $8 \%$ under the age of 40 had pathogenic variants and these were in BRCA1, TP53 and CHEK2. These figures rise to $16 \%$ and $11 \%$, respectively, if only women with a family history of breast cancer are considered, Table 5 . In contrast, the frequency of pathogenic variants in ER-positive DCIS under the age of 40 was much higher; $9 \%$ of women under the age of 50 had pathogenic variants rising to $29 \%$ under the age of $40(14 \%$ and $42 \%$, respectively, if only women with a family history of breast cancer are considered) Table 6.

\section{Conclusions}

This study has shown that a DCIS-associated malignant pathway can occur in patients who have pathogenic variants in BRCA2, CHEK2, PALB2, BRCA1 and TP53. We also show that the focus of genetic testing should be on ER-positive, intermediate-, and high-grade DCIS from patients under the age of 40, rather than ER-negative DCIS, although restricting such testing to those age under 40 would fail to identify the majority of $C H E K 2$ and PALB2 mutation carriers. Once mutations are identified in these women, chemoprevention with tamoxifen and surveillance is a potential alternative to prophylactic mastectomy, particularly in CHEK2 carriers where the risk of invasive disease is less. Further studies with

Table 6 Frequency of pathogenic variants in ER-positive DCIS by age

\begin{tabular}{|c|c|c|c|c|}
\hline Gene & $\begin{array}{l}\text { Frequency under } 40 \text { years } \\
N=45\end{array}$ & $\begin{array}{l}\text { Frequency under } 40 \text { years with } \\
\mathrm{FH} \mathrm{N}=24\end{array}$ & $\begin{array}{l}\text { Frequency under } 50 \text { years } N \\
=397\end{array}$ & $\begin{array}{l}\text { Frequency under } 50 \text { years } \\
\text { with FH } \\
N=197\end{array}$ \\
\hline BRCA2 & $8(17.8 \%)$ & $7(29.2 \%)$ & $16(4 \%)$ & $14(7.1 \%)$ \\
\hline CHEK2 & $2(4.4 \%)$ & 1 (4.2\%) & $12(3 \%)$ & 7 (3.6\%) \\
\hline PALB2 & 1 (2.2\%) & $1(4.2 \%)$ & $5(1.3 \%)$ & $4(2 \%)$ \\
\hline$B R C A 1$ & 1 (2.2\%) & $0(0 \%)$ & $1(0.3 \%)$ & $0(0 \%)$ \\
\hline TP53 & 1 (2.2\%) & 1 (4.2\%) & $2(0.5 \%)$ & $2(1 \%)$ \\
\hline $\begin{array}{l}\text { Total (\% of ER+ } \\
\text { cases) }\end{array}$ & $\begin{array}{l}13 \text { variants }(29 \%) \text { in } 11 \text { women } \\
(24 \%)^{*}\end{array}$ & $\begin{array}{l}10 \text { variants (42\%) in } 9 \text { women } \\
(38 \%)\end{array}$ & $\begin{array}{l}36 \text { variants (9\%) in } 32 \\
\text { women (8\%) }\end{array}$ & $\begin{array}{l}27 \text { variants }(14 \%) \text { in } 24 \\
\text { women }(12 \%)\end{array}$ \\
\hline
\end{tabular}

$\mathrm{FH}$ family history of breast cancer in any relative

*Some individuals carry 2 variants 
long-term follow-up data are required to ascertain whether these germline pathogenic variants identify a subgroup of DCIS that are more likely to progress to invasive disease or whether somatic changes in the DCIS are a more important predictor of recurrence.

\section{Additional files}

Additional file 1: Targeted sequencing panel. (DOCX $19 \mathrm{~kb}$ )

Additional file 2: Coverage data for all pathogenic variants identified. (XLSX $14 \mathrm{~kb}$ )

Additional file 3: Definition of variants. (DOCX $18 \mathrm{~kb})$

Additional file 4: Amplicons that failed to amplify consistently. (DOCX $19 \mathrm{~kb})$

Additional file 5: Copy number variation detected by ONCOCNV.

(DOCX $15 \mathrm{~kb}$ )

Additional file 6: $B R C A 2$ pathogenic variants in cases. (DOCX $21 \mathrm{~kb}$ )

Additional file 7: CHEK2 pathogenic variants in cases. (DOCX $20 \mathrm{~kb}$ )

Additional file 8: PALB2 pathogenic variants in cases. (DOCX $19 \mathrm{~kb}$ )

Additional file 9: $B R C A 1$ pathogenic variants in cases. (DOCX $18 \mathrm{~kb}$ )

Additional file 10: a: TP53 pathogenic variants in cases. b: TP53 VUS in cases. (DOCX $20 \mathrm{~kb}$ )

Additional file 11: Age of onset of DCIS and family history details of women with two pathogenic variants in different genes. (DOCX 19 kb)

Additional file 12: Frequency of variants of unknown significance and DCIS in women $<50$ years of age by gene. (DOCX $19 \mathrm{~kb}$ )

\section{Abbreviations}

$95 \mathrm{Cl}$ : 95\% confidence interval; DCIS: Ductal carcinoma in situ; IDC: Invasive ductal cancer.; ILC: Invasive lobular cancer; LCIS: Lobular carcinoma in situ; OR: Odds ratio; VUS: Variant of unknown significance

\section{Acknowledgements}

We thank all the individuals who took part in this study and all the clinicians, technicians and administrative staff who have enabled this work to be carried out. In particular, we thank Maria Troy, Kelly Kohut and Pat Gorman.

\section{Funding}

This work was supported by a King's Health Partner's Research and Development Challenge Fund (R150505), a Breast Cancer Campaign Project Grant (2011NovPR49) and the National Institute for Health Research (NIHR) Biomedical Research Centre based at Guy's and St Thomas' NHS Foundation Trust and King's College London. The views expressed are those of the author(s) and not necessarily those of the NHS, the NIHR or the Department of Health. Sample collection was funded by a CRUK Project grant (8873).

\section{Availability of data and materials}

The dataset supporting the conclusions of this article can be found in the additional data (Additional file 2).

\section{Authors' contributions}

EJS conceived the study and wrote the manuscript. CP performed all the next-generation sequencing, under the supervision of MAS. CP and IA performed the analysis, under the supervision of MAS. VS performed the Sanger sequencing. CM, AM, AC and CG collected and provided samples and questionnaire data. SP reviewed histology of samples. AMeg performed the copy number analysis. EJS, IT and RR set up the GLACIER and ICICLE studies. All authors reviewed the manuscript. All authors read and approved the final manuscript.

\section{Ethics approval and consent to participate}

All patients and controls were recruited through the following ethically approved studies and gave consent to genetic testing (GLACIER study, MREC
06/Q1702/64; ICICLE, MREC 08/H0502/4; King's Health Partners breast tissue bank, NHS REC ref. 12-EE-0493).

\section{Consent for publication}

Not applicable.

\section{Competing interests}

The authors declare that there is no competing interests.

\section{Publisher's Note}

Springer Nature remains neutral with regard to jurisdictional claims in published maps and institutional affiliations.

\section{Author details}

${ }^{1}$ School of Cancer and Pharmaceutical Sciences, Guy's Hospital, King's College London, London SE1 9RT, UK. ${ }^{2}$ Medical and Molecular Genetics, Guy's Hospital, King's College London, London SE1 9RT, UK. ${ }^{3}$ Institute of Cancer and Genomic Sciences, University of Birmingham, Edgbaston, Birmingham B15 2TT, UK. ${ }^{4}$ Department of Oncology, UCLH Foundation Trust, London NW1 2PG, UK. Innovation Hub, Guy's Cancer Centre, Guy's Hospital, London SE1 9RT, UK.

Received: 6 September 2018 Accepted: 17 April 2019

Published online: 06 May 2019

References

1. Wong H, Lau S, Yau T, Cheung P, Epstein RJ. Presence of an in situ component is associated with reduced biological aggressiveness of sizematched invasive breast cancer. Br J Cancer. 2010;102(9):1391-6.

2. Ruszczyk M, Zirpoli G, Kumar S, Bandera EV, Bovbjerg DH, Jandorf L, Khoury T, Hwang H, Ciupak G, Pawlish K, et al. Breast cancer risk factor associations differ for pure versus invasive carcinoma with an in situ component in casecontrol and case-case analyses. Cancer Cause Control. 2016;27(2):183-98.

3. Kim SY, Jung SH, Kim MS, Baek IP, Lee SH, Kim TM, Chung YJ, Lee SH. Genomic differences between pure ductal carcinoma in situ and synchronous ductal carcinoma in situ with invasive breast cancer. Oncotarget. 2015;6(10):7597-607.

4. Wong H, Lau S, Leung R, Chiu J, Cheung P, Wong TT, Liang R, Epstein RJ, Yau T. Coexisting ductal carcinoma in situ independently predicts lower tumor aggressiveness in node-positive luminal breast cancer. Med Oncol. 2012;29(3):1536-42.

5. Li Cl, Daling JR, Malone KE. Age-specific incidence rates of in situ breast carcinomas by histologic type, 1980 to 2001. Cancer Epidem Biomar. 2005; 14(4):1008-11.

6. Leonard GD, Swain SM. Ductal carcinoma in situ, complexities and challenges. J Natl Cancer Inst. 2004;96(12):906-20.

7. Claus EB, Stowe M, Carter D. Breast carcinoma in situ: risk factors and screening patterns. J Natl Cancer Inst. 2001;93(23):1811-7.

8. Reeves GK, Pirie K, Green J, Bull D, Beral V, Million Women Study C. Comparison of the effects of genetic and environmental risk factors on in situ and invasive ductal breast cancer. Int J Cancer. 2012;131(4):930-7.

9. Claus EB, Stowe M, Carter D. Family history of breast and ovarian cancer and the risk of breast carcinoma in situ. Breast Cancer Res Treat. 2003;78(1): 7-15.

10. Kerlikowske K, Barclay J, Grady D, Sickles EA, Ernster V. Comparison of risk factors for ductal carcinoma in situ and invasive breast cancer. J Natl Cancer Inst. 1997;89(1):76-82.

11. Petridis C, Brook MN, Shah V, Kohut K, Gorman P, Caneppele M, Levi D, Papouli E, Orr N, Cox A, et al. Genetic predisposition to ductal carcinoma in situ of the breast. Breast Cancer Res. 2016;18(1):22.

12. Claus EB, Petruzella S, Matloff E, Carter D. Prevalence of BRCA1 and BRCA2 mutations in women diagnosed with ductal carcinoma in situ. JAMA. 2005; 293(8):964-9.

13. Hall MJ, Reid JE, Wenstrup RJ. Prevalence of BRCA1 and BRCA2 mutations in women with breast carcinoma in situ and referred for genetic testing. Cancer Prev Res. 2010;3(12):1579-85.

14. Lester SC, Bose S, Chen YY, Connolly JL, de Baca ME, Fitzgibbons PL, Hayes DF, Kleer C, O'Malley FP, Page DL, et al. Protocol for the examination of specimens from patients with invasive carcinoma of the breast. Arch Pathol Lab Med. 2009;133(10):1515-38. 
15. Hintzsche JD, Robinson WA, Tan AC. A survey of computational tools to analyze and interpret whole exome sequencing data. Int J Genomics. 2016; 2016:7983236

16. Talevich E, Shain AH, Botton T, Bastian BC. CNVkit: genome-wide copy number detection and visualization from targeted DNA sequencing. PLoS Comput Biol. 2016;12(4):e1004873.

17. Boeva V, Popova T, Lienard M, Toffoli S, Kamal M, Le Tourneau C, Gentien D, Servant N, Gestraud P, Rio Frio T, et al. Multi-factor data normalization enables the detection of copy number aberrations in amplicon sequencing data. Bioinformatics. 2014;30(24):3443-50.

18. Chuba PJ, Hamre MR, Yap J, Severson RK, Lucas D, Shamsa F, Aref A. Bilateral risk for subsequent breast cancer after lobular carcinoma-in-situ: analysis of surveillance, epidemiology, and end results data. J Clin Oncol. 2005;23(24):5534-41.

19. Olivier M, Eeles $R$, Hollstein M, Khan MA, Harris CC, Hainaut P. The IARC TP53 database: new online mutation analysis and recommendations to users. Hum Mutat. 2002;19(6):607-14.

20. Mersch J, Brown N, Pirzadeh-Miller S, Mundt E, Cox HC, Brown K, Aston M, Esterling L, Manley S, Ross T. Prevalence of variant reclassification following hereditary cancer genetic testing. JAMA. 2018;320(12):1266-74.

21. Olivier $M$, Hollstein $M$, Hainaut P. TP53 mutations in human cancers: origins, consequences, and clinical use. Cold Spring Harb Perspect Biol. 2010;2(1): a001008.

22. Bouaoun L, Sonkin D, Ardin M, Hollstein M, Byrnes G, Zavadil J, Olivier M. TP53 variations in human cancers: new lessons from the IARC TP53 database and genomics data. Hum Mutat. 2016;37(9):865-76.

23. Yang RL, Mick R, Lee K, Graves HL, Nathanson KL, Domchek SM, Kelz RR, Zhang PJ, Czerniecki BJ. DCIS in BRCA1 and BRCA2 mutation carriers: prevalence, phenotype, and expression of oncodrivers C-MET and HER3. J Transl Med. 2015;13:335.

24. Krammer J, Pinker-Domenig K, Robson ME, Gonen M, Bernard-Davila B, Morris EA, Mangino DA, Jochelson MS. Breast cancer detection and tumor characteristics in BRCA1 and BRCA2 mutation carriers. Breast Cancer Res Treat. 2017;163(3):565-71.

25. Aloraifi F, Alshehhi M, McDevitt T, Cody N, Meany M, O'Doherty A, Quinn CM, Green AJ, Bracken A, Geraghty JG. Phenotypic analysis of familial breast cancer: comparison of BRCAx tumors with BRCA1-, BRCA2-carriers and nonfamilial breast cancer. Eur J Surg Oncol. 2015;41(5):641-6.

26. Schmidt MK, Hogervorst F, van Hien R, Cornelissen S, Broeks A, Adank MA, Meijers H, Waisfisz Q, Hollestelle A, Schutte M, et al. Age- and tumor subtype-specific breast cancer risk estimates for CHEK2*1100delC carriers. J Clin Oncol. 2016;34(23):2750-60.

27. Couch FJ, Shimelis H, Hu C, Hart SN, Polley EC, Na J, Hallberg E, Moore R, Thomas A, Lilyquist J, et al. Associations between cancer predisposition testing panel genes and breast cancer. JAMA Oncol. 2017;3(9):1190-6.

28. Teo ZL, Provenzano E, Dite GS, Park DJ, Apicella C, Sawyer SD, James PA, Mitchell G, Trainer AH, Lindeman GJ, et al. Tumour morphology predicts PALB2 germline mutation status. Br J Cancer. 2013;109(1):154-63.

29. Antoniou AC, Casadei S, Heikkinen T, Barrowdale D, Pylkas K, Roberts J, Lee A, Subramanian D, De Leeneer K, Fostira F, et al. Breast-cancer risk in families with mutations in PALB2. N Engl J Med. 2014;371(6):497-506.

30. Masciari S, Dillon DA, Rath M, Robson M, Weitzel JN, Balmana J, Gruber SB, Ford JM, Euhus D, Lebensohn A, et al. Breast cancer phenotype in women with TP53 germline mutations: a Li-Fraumeni syndrome consortium effort. Breast Cancer Res Treat. 2012;133(3):1125-30.

31. Evans DG, Moran A, Hartley R, Dawson J, Bulman B, Knox F, Howell A, Lalloo F. Long-term outcomes of breast cancer in women aged 30 years or younger, based on family history, pathology and BRCA1/BRCA2/TP53 status. Br J Cancer. 2010;102(7):1091-8.

32. Bougeard G, Renaux-Petel M, Flaman J-M, Charbonnier C, Fermey P, Belott M, Gauthier-Villars M, Stoppa-Lyonnet D, Consolino E, Brugières L, et al. Revisiting Li-Fraumeni syndrome from TP53 mutation carriers. J Clin Oncol. 2015;33(21):2345-52.

33. Schmidt MK, van den Broek AJ, Tollenaar RA, Smit VT, Westenend PJ, Brinkhuis M, Oosterhuis WJ, Wesseling J, Janssen-Heijnen ML, Jobsen JJ, et al. Breast cancer survival of BRCA1/BRCA2 mutation carriers in a hospitalbased cohort of young women. J Natl Cancer Inst. 2017;109(8):djw329. https://doi.org/10.1093/jnci/djw329.

\section{Ready to submit your research? Choose BMC and benefit from:}

- fast, convenient online submission

- thorough peer review by experienced researchers in your field

- rapid publication on acceptance

- support for research data, including large and complex data types

- gold Open Access which fosters wider collaboration and increased citations

- maximum visibility for your research: over $100 \mathrm{M}$ website views per year

At BMC, research is always in progress.

Learn more biomedcentral.com/submissions 